\title{
Infraorbital nerve: a surgically relevant landmark for the pterygopalatine fossa, cavernous sinus, and anterolateral skull base in endoscopic transmaxillary approaches
}

\author{
Ali M. Elhadi, MD, PhD, ${ }^{1}$ Hasan A. Zaidi, MD, ${ }^{1}$ Kaan Yagmurlu, MD, ${ }^{1}$ Shah Ahmed, BS, ${ }^{1}$ \\ Albert L. Rhoton Jr., MD, ${ }^{2}$ Peter Nakaji, MD, ${ }^{1}$ Mark C. Preul, MD, ${ }^{1}$ and Andrew S. Little, MD ${ }^{1}$ \\ 'Department of Neurosurgery, Barrow Neurological Institute, St. Joseph's Hospital and Medical Center, Phoenix, Arizona; and \\ 2Department of Neurological Surgery, University of Florida, Gainesville, Florida
}

\begin{abstract}
OBJECTIVE Endoscopic transmaxillary approaches (ETMAs) address pathology of the anterolateral skull base, including the cavernous sinus, pterygopalatine fossa, and infratemporal fossa. This anatomically complex region contains branches of the trigeminal nerve and external carotid artery and is in proximity to the internal carotid artery. The authors postulated, on the basis of intraoperative observations, that the infraorbital nerve (ION) is a useful surgical landmark for navigating this region; therefore, they studied the anatomy of the ION and its relationships to critical neurovascular structures and the maxillary nerve (V2) encountered in ETMAs.
\end{abstract}

METHODS Endoscopic anatomical dissections were performed bilaterally in 5 silicone-injected, formalin-fixed cadaveric heads (10 sides). Endonasal transmaxillary and direct transmaxillary (Caldwell-Luc) approaches were performed, and anatomical correlations were analyzed and documented. Stereotactic imaging of each specimen was performed to correlate landmarks and enable precise measurement of each segment.

RESULTS The ION was readily identified in the roof of the maxillary sinus at the beginning of the surgical procedure in all specimens. Anatomical dissections of the ION and the maxillary branch of the trigeminal nerve (V2) to the cavernous sinus suggested that the ION/V2 complex has 4 distinct segments that may have implications in endoscopic approaches: 1) Segment I, the cutaneous segment of the ION and its terminal branches (5-11 branches) to the face, distal to the infraorbital foramen; 2) Segment II, the orbitomaxillary segment of the ION within the infraorbital canal from the infraorbital foramen along the infraorbital groove (length $12 \pm 3.2 \mathrm{~mm}$ ); 3) Segment III, the pterygopalatine segment within the pterygopalatine fossa, which starts at the infraorbital groove to the foramen rotundum $(13 \pm 2.5 \mathrm{~mm})$; and 4) Segment IV, the cavernous segment from the foramen rotundum to the trigeminal ganglion $(15 \pm 4.1 \mathrm{~mm})$, which passes in the lateral wall of the cavernous sinus. The relationship of the ION/V2 complex to the contents of the cavernous sinus, carotid artery, and pterygopalatine fossa is described in the text.

CONCLUSIONS The ION/V2 complex is an easily identifiable and potentially useful surgical landmark to the foramen rotundum, cavernous sinus, carotid artery, pterygopalatine fossa, and anterolateral skull base during ETMAs.

http://thejns.org/doi/abs/10.3171/2015.9.JNS151099

KEY WORDS anatomical study; anatomy; endoscopic surgery; infraorbital nerve; pterygopalatine fossa; skull base

$\mathrm{E}$ XPANDED endoscopic surgery is a standard option in the armamentarium for skull base approaches to address numerous pathologies..$^{2,4,13,16,27}$ Technical improvements over the past decade have made endoscopy a viable alternative to conventional microscopic or open procedures. ${ }^{10,31}$ Expanded endoscopic endonasal approaches have been used to access lesions in the clivus, ${ }^{17,20}$ posterior fossa, ${ }^{18,29}$ craniovertebral junction,, 24 and anterior fossa. ${ }^{29}$ One such expanded endoscopic approach, the endoscopic transmaxillary approach (ETMA), is used to access lesions within the maxillary sinus, lateral sphenoid sinus, cavernous sinus, and anterolateral skull base. ${ }^{1,8,14,23,25,32,34}$ The transmaxillary corridor is most commonly accessed through an endonasal approach and a medial maxillectomy or through a sublabial incision and a direct anterior maxillectomy (i.e., Caldwell-Luc approach) ${ }^{8,23,25}$

Endoscopic approaches to the anterolateral skull base are challenging for neurosurgeons because of the unfamil-

ABBREVIATIONS ETMA = endoscopic transmaxillary approach; ICA = internal carotid artery; IOA = infraorbital artery; ION = infraorbital nerve.

SUBMITTED May 18, 2015. ACCEPTED September 10, 2015.

INCLUDE WHEN CITING Published online March 4, 2016; DOI: 10.3171/2015.9.JNS151099. 
iar ventral anatomical perspective and the complex critical regional anatomy of the pterygopalatine and infratemporal fossae. ${ }^{6,32,33}$ Because space-occupying lesions within this region can distort normal anatomy and stereotactic image guidance can be misleading, there is a need for reliable anatomical landmarks during surgery. The infraorbital nerve (ION) is a sensory branch of the trigeminal nerve that travels in the roof of the maxillary sinus to innervate the skin of the cheek. Our group has found that the ION is readily identifiable early in the ETMA and that it is potentially useful as an anatomical reference as dissection proceeds deeper into the skull base. Therefore, we sought to study the ION and its anatomical relationships to determine its potential utility as an endoscopic surgical landmark. We also sought to define its relationship to critical anatomical structures, such as the carotid artery, the cavernous sinus, and the contents of the pterygopalatine and infratemporal fossae. In this study, we also sought to define the ION/maxillary nerve (V2) complex and identify its 4 segments as a useful surgical road map.

\section{Methods}

One formalin-fixed, silicone-injected cadaveric head was dissected using conventional dissection techniques for illustrative purposes to track the course of the maxillary nerve, as previously described ${ }^{35}$ Separately, 5 formalin-fixed, silicone-injected cadaveric heads without any known intracranial, extracranial, or sinonasal pathology were examined using an endoscope under stereotactic guidance for all anatomical measurements. In the remainder of this paper, we focus on the methods of these 5 fixed cadaveric dissections.

Endoscopic dissections were performed bilaterally (10 sides). Cadaveric specimens were fixed in the supine position using a Mayfield head holder. For visualization, we used a standard endoscopic unit with a high-definition camera with 3 charge-coupled devices (3-CCD camera; Karl Storz Endoscopy-America, Inc.) and an 18-cm-long, 4-mm-diameter, $0^{\circ}$ Hopkins II rod-lens endoscope connected to a fiberoptic cable as a light source. Dissections were performed using standard straight and curved endoscopic instrumentations (Karl Storz); angular visualization was enabled using $30^{\circ}$ and $45^{\circ}$ endoscopes. Other instrumentation used to assist with dissection included dissectors, scissors (curved and straight), Kerrison rongeurs, and pneumatic drills.

Prior to dissection, high-resolution stereotactic imaging of each specimen was performed. Images were then uploaded on an image guidance platform (StealthStation Treon Plus with FrameLink software, Medtronic, PLC) to ensure the proper trajectory and to assist with identifying anatomical structures, correlations, and measurements. As previously described by our group, image guidance was used to obtain anatomical measurements and distances between important neuroanatomical structures and to confirm the anatomy. ${ }^{9}$

Endoscopic endonasal transmaxillary and endoscopic sublabial transmaxillary transpterygoid approaches were performed on both sides, as described elsewhere by us and others. ${ }^{1,8,32,34}$ In most specimens, a combined approach was performed to enable wider dissection and a more detailed view of the ION and the surrounding structures.

\section{Exposure of the ION to the Cavernous Sinus}

After performing the maxillary antrostomy, identifying the ION in the roof of the maxillary sinus, and removing the posterior wall of the maxillary sinus, we exposed the retromaxillary space in the following fashion: the pterygopalatine ganglia were identified within the pterygopalatine fossa by simply following the ION as it curves medially to become the maxillary branch of the trigeminal nerve (V2). After complete removal of the posterior maxillary wall, branches from the V2 that are directed inferiorly to the pterygopalatine ganglia could be identified a few millimeters before the foramen rotundum. The sphenoid bone was then drilled to expose the V2 and the anterior wall of the cavernous sinus. The pterygoid process and the pterygoid wedge (i.e., the bone between the foramen rotundum and the vidian canal) were drilled to expose the V2 as it enters the lateral wall of the cavernous sinus, dividing the lateral wall into the anteromedial triangle (between V2 and the ophthalmic nerve, V1) and the anterolateral triangle (between V2 and the mandibular nerve, V3). The vidian canal was identified and drilled circumferentially to expose the anterior genu of the carotid artery. ${ }^{22}$

\section{Exposure of the Mandibular Branch of the Trigeminal Nerve (V3) to the Cavernous Sinus}

To expose the V3 within the infratemporal fossa, we conducted further dissection posteriorly and laterally. The cartilaginous portion of the eustachian tube was preserved, and the V3 was identified. Kerrison rongeurs were then used to remove the thin plate of sphenoid bone to open the foramen ovale, thereby exposing the inferior recess of the lateral wall of the cavernous sinus.

\section{Results}

Anatomical dissections suggested that the ION in the roof of the maxillary sinus serves as a useful superficial landmark for identifying deeper elements, including the V2, the pterygopalatine fossa, and the cavernous sinus. The cutaneous branches, ION, and V2 form the ION/V2 complex, which we have conceptualized into 4 segments (Fig. 1). These segments-cutaneous, orbitomaxillary, pterygopalatine, and cavernous-are numbered from I to IV, from superficial to deep, in the order that they are approached surgically. Segment I, the cutaneous segment, represents the terminal end of the ION and the terminal branches of the ION (5-11 branches). Segment I begins in the infraorbital foramen and terminates in the numerous cutaneous branches. Segment II, the orbitomaxillary segment, represents the ION after leaving the pterygopalatine fossa as it travels through the infraorbital groove through the infraorbital canal and ends at the infraorbital foramen in the superior one-third of the anterior maxillary wall, just below the orbit (length $12 \pm 3.2 \mathrm{~mm}$ ). Segment III, the pterygopalatine segment, resides within the pterygopalatine fossa from the foramen rotundum to the infraorbital groove, just below the superior orbital fissure (length $13 \pm$ $2.5 \mathrm{~mm}$ ). It contains the proximal portion of the ION and the distal end of the V2 trunk. Segment IV, the cavernous 


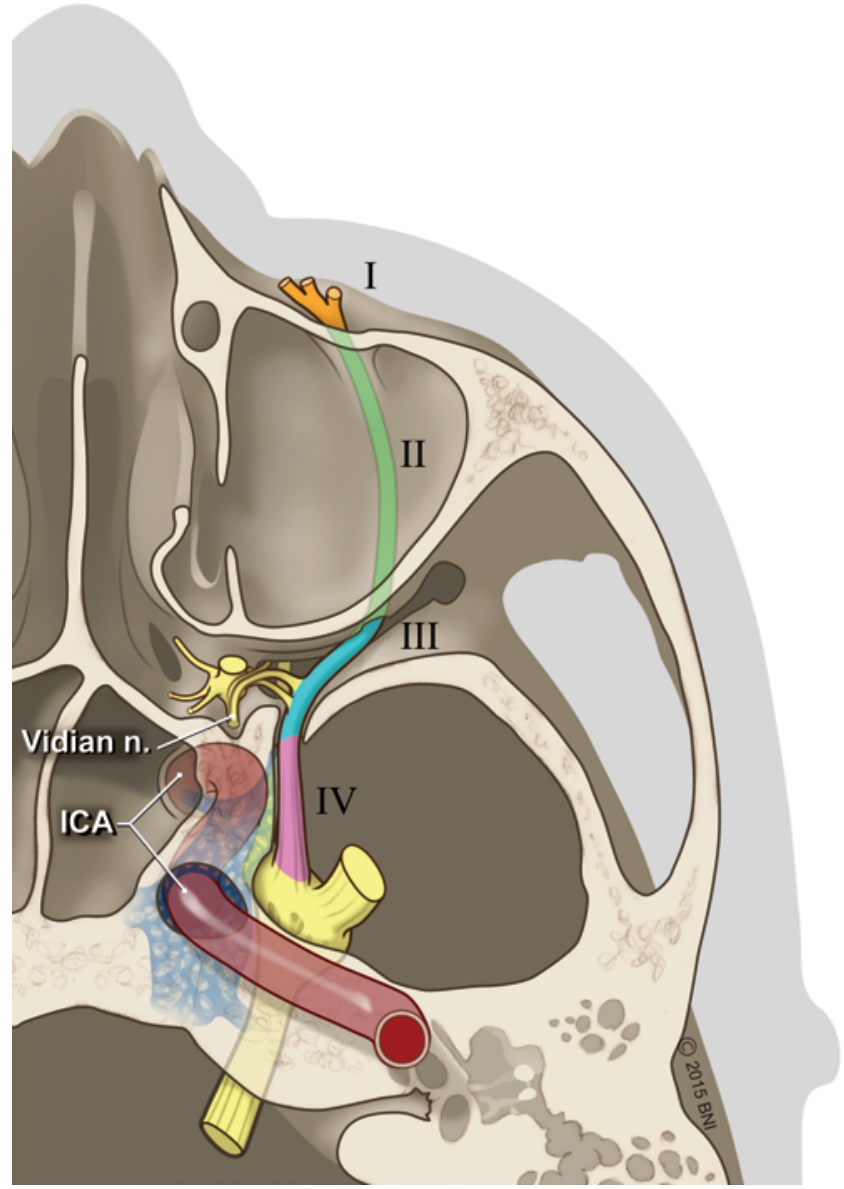

FIG. 1. Illustration showing the 4 distinct anatomical segments of the ION and the V2 in the order that they would be approached surgically: cutaneous (Segment I, orange), orbitomaxillary (Segment II, green), pterygopalatine (Segment III, turquoise), and cavernous (Segment IV, pink). ICA = internal carotid artery; $\mathrm{n}=$ nerve. Used with permission from Barrow Neurological Institute, Phoenix, Arizona. Figure is available in color online only.

segment, starts at the trigeminal ganglion as the V2 exits the ganglion within the nerve sheath to the foramen rotundum (length $15.0 \pm 4.1 \mathrm{~mm}$ ).

\section{Segment l: Cutaneous Segment}

The terminal end of the ION exits the infraorbital foramen, which was located in the middle of the upper onethird of the anterior wall of the maxillary sinus in $3(60 \%)$ of the 5 specimens ( 6 of 10 specimen sides) and more laterally in 2 specimens (40\%). After emerging from the infraorbital foramen, the ION terminates in 5-11 terminal branches (Fig. 2). These cutaneous branches innervate the skin of the midface, with 3-9 endings that branch medially. The largest branch is the superior labial branch, which tends to be the more inferior of the medial branches. The second largest branch is the external nasal branch, which supplies the skin of the lower nose. Additional branches include the internal nasal branch, which supplies the nasal septum, and the inferior palpebral nerve, which innervates the skin and conjunctiva of the lower eyelid. The remaining branches of the ION supply different areas of the skin

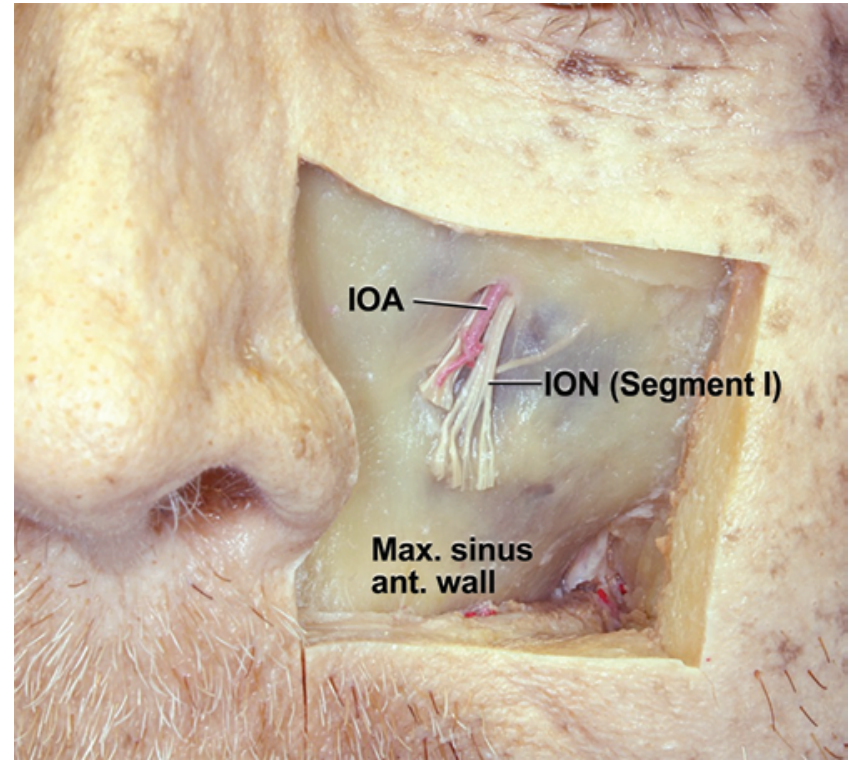

FIG. 2. The cutaneous segment (Segment I) of the left ION. This segment is the terminus of the ION, and it typically contains 5-11 terminal branches. Max. sinus ant. = maxillary sinus anterior. Reproduced with permission from the Rhoton Collection (http://rhoton.ineurodb.org/), CC BY-NC-SA 4.0 (http://creativecommons.org/licenses/by-nc-sa/4.0/). Figure is available in color online only.

of the midface from the nose medially to the anterior onethird of the zygoma. From below the orbit to the upper lip are branches that arise directly from the ION or from the main 4 terminal branches. In our specimens, the infraorbital artery (IOA) exited the infraorbital foramen along with the ION within the same sheath in $7(88 \%)$ of 8 specimen sides, while in 1 side (12\%) the IOA emerged separately from the nerve. In 2 sides, the relationship of the IOA and ION was indeterminate. The IOA then emerges between the internal nasal branch and the inferior palpebral branch to become more superficial to the terminal ION, and it terminates with feeding branches that supply the 4 main branches of the ION.

From an endoscopic perspective, locations of the terminal branches are most relevant during a sublabial direct antrostomy. With the direct transmaxillary approach, a periosteal elevator is used to create a plane just under these branches to avoid injury to the nerves..$^{12}$ These cutaneous branches are not encountered in an endonasal transmaxillary approach unless it is necessary to perform wider bony removal of the anterior medial maxillary wall and the medial anterior maxillary wall, such as in an endoscopic Denker's approach. ${ }^{19}$

\section{Segment II: Orbitomaxillary Segment}

The orbitomaxillary segment of the maxillary nerve represents the ION within the superior maxillary wall/ orbital floor (Fig. 3). The second segment of the ION/V2 complex was easily identified in the roof of the maxillary sinus in all 10 sides, which is consistent with our intraoperative impression. In most surgical cases, this point is where the ION is first identified. It begins in the infraorbital groove at the level of the posterior maxillary wall and travels to the distal end of the infraorbital canal. Identifi- 


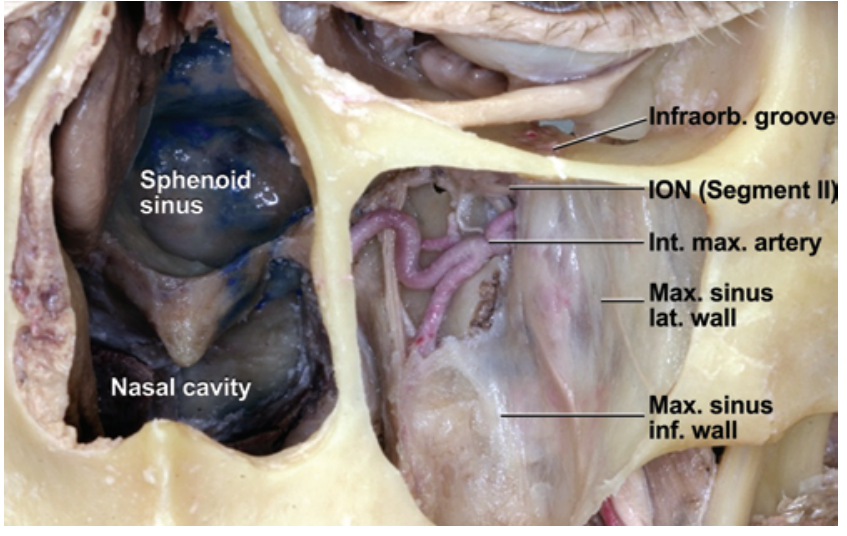

FIG. 3. The orbitomaxillary segment (Segment II) of the left ION. This segment of the ION travels in the infraorbital (Infraorb.) groove, located in the superior wall of the maxillary sinus. Int. max. = internal maxillary; Max. sinus inf. = maxillary sinus inferior; Max. sinus lat. = maxillary sinus lateral. Reproduced with permission from the Rhoton Collection (http://rhoton.ineurodb.org/), CC BY-NC-SA 4.0 (http://creativecommons. org/licenses/by-nc-sa/4.0/). Figure is available in color online only.

cation of the ION can be aided by removing the maxillary sinus mucosa, but this is often unnecessary. The IOA is the lateral terminal branch of the internal maxillary artery, which then curves medially toward the ION to stay lateral to the nerve. Just before accompanying the ION along the infraorbital groove, the artery loops from underneath the nerve to become medial to the nerve. The IOA is located medially to the ION throughout the entire segment.

From the orbitomaxillary segment, 2 main alveolar branches, the middle superior and the anterior superior, are produced. These branches curve and descend on the maxillary tuberosity and supply the incisors, canines, molar teeth, and parts of the gum and mucosa. In all of our specimens, the anterior superior alveolar branch emerged within the infraorbital canal, while the middle superior alveolar branches of the ION emerged in the infraorbital groove in 50\% $(n=5)$ of the specimen sides and in the pterygopalatine fossa in $50 \%(\mathrm{n}=5)$ of the sides.

During the ETMA, the ION can assist with anatomical orientation (Figs. 4 and 5). With the use of a $0^{\circ}$ endoscope, the ION can be fixed at the 1 o'clock position in the endoscopic view, thus enabling a complete view of the posterior maxillary wall, the greater sphenopalatine nerve, and the sphenopalatine artery.

\section{Segment III: Pterygopalatine Segment}

The pterygopalatine segment of the maxillary nerve represents the termination of the V2 and the ION within the pterygopalatine fossa (Fig. 6). It extends from the foramen rotundum proximally to the infraorbital groove distally. The maxillary nerve exits the foramen rotundum, then produces the pterygopalatine branches in the pterygopalatine ganglion. Beyond that point, it continues as the ION. Branching of the zygomatic nerve occurred within the foramen in 5 specimen sides $(50 \%)$. Thus, the zygomatic nerve usually emerges from the foramen as a separate nerve from the V2.

Our results were not conclusive as to whether the posterior alveolar branch emerges from the ION or from the ter-

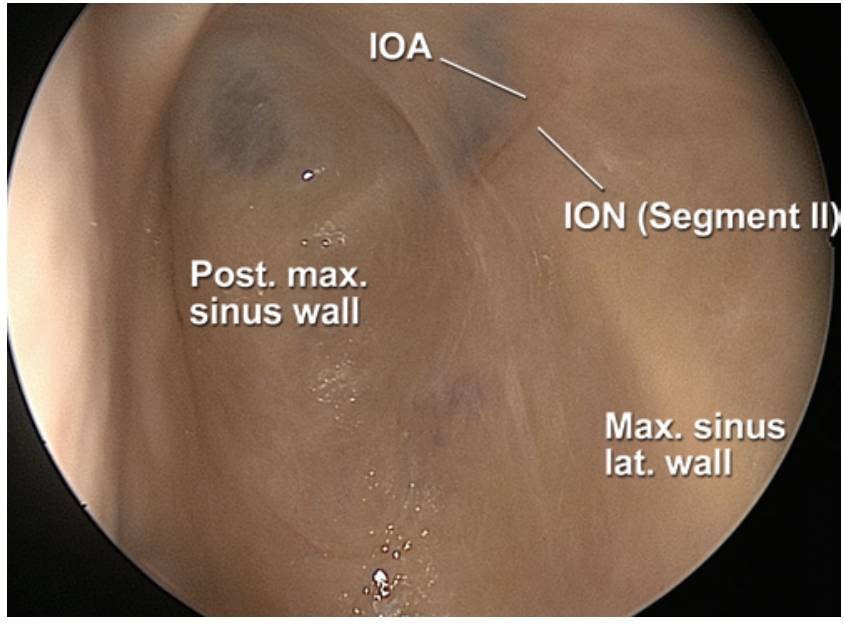

FIG. 4. Endoscopic endonasal view of the orbitomaxillary segment (Segment II) of the maxillary nerve via a $0^{\circ}$ endoscope. The maxillary sinus has been entered, and the ION is visible through the thin bony plate of the superior maxillary sinus wall after removal of the sinus mucosa. The IOA is located medially to the ION throughout the entire segment. Max. sinus lat. $=$ maxillary sinus lateral; Post. $\max .=$ posterior maxillary. Reproduced with permission from the Rhoton Collection (http://rhoton. ineurodb.org/), CC BY-NC-SA 4.0 (http://creativecommons.org/licenses/ by-nc-sa/4.0/). Figure is available in color online only.

minal V2. In 5 specimen sides (50\%), the posterior superior alveolar nerve emerged as 2 separate nerves from the inferior surface of the V2 and/or the ION, and it descended along the posterior maxillary wall to supply the posterior part of the gum and mucosa. In the other 5 (50\%) specimen sides, the posterior superior alveolar nerve branched off as a single trunk from the inferior surface of the V2.

From an endoscopic perspective, the ION can be tracked backward through a transmaxillary approach after removal of the posterior maxillary wall to expose the contents of the pterygopalatine fossa (Figs. 5-7). Branches from the V2 to the pterygopalatine ganglia then join the ION to form the V2. Keeping the ION in the 1 o'clock position in the endoscopic view enables complete visualization of the contents of the pterygopalatine fossa, including the pterygopalatine ganglion, which can be seen directly in the center of the operative field. Segment III of the ION/ V2 complex is a useful surgical landmark for separating the pterygopalatine fossa from the infratemporal fossa. It is also useful as a landmark when used in conjunction with the vidian canal to identify the entire anterior face of the cavernous sinus and to skeletonize the carotid artery. The petrous carotid artery lies inferior and posterior to the foramen rotundum, and the cavernous internal carotid artery (ICA) resides medial to the foramen rotundum (Figs. $8-10)$. With such an exposure, a large window into the cavernous sinus can be created.

\section{Segment IV: Cavernous Segment}

The cavernous segment of the maxillary nerve represents that portion of the nerve located within the cavernous sinus bounded anteriorly by the foramen rotundum (Figs. 8-10). Segment IV of the ION/V2 complex extends from the beginning of the V2 as it branches from the trigeminal nerve at the edge of Meckel's cave and enters 


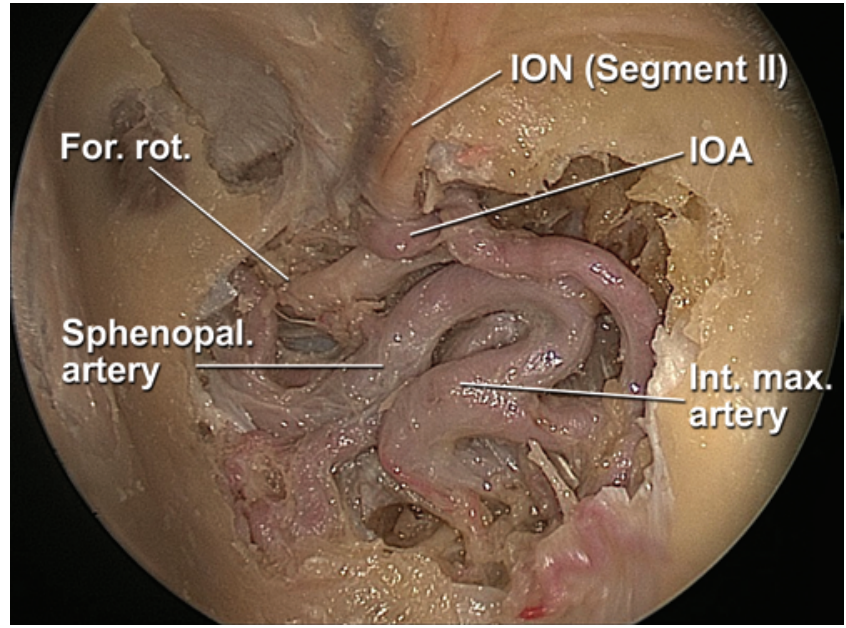

FIG. 5. Endoscopic endonasal view of the pterygopalatine segment (Segment III) of the left ION via a $0^{\circ}$ endoscope. After the posterior wall of the maxillary sinus is removed, the ION (Segment II) can be followed back to the pterygopalatine fossa as it emerges from the foramen rotundum (For. rot.). The IOA loops underneath and travels medial to the ION. Int. max. = internal maxillary; Sphenopal. $=$ sphenopalatine. Figure is available in color online only.

the cavernous sinus within the lateral wall at the level of the posterior bend of the ICA. The V2 divides the lateral wall into the anteromedial triangle, between the V2 and the ophthalmic nerve (V1), and the anterolateral triangle, between the V2 and the mandibular nerve (V3). The V2 then continues to travel anterior, to exit the cavernous sinus through the inferior-anterior recess of the lateral wall and to exit the middle cranial fossa through the foramen rotundum.

The cavernous segment of the ICA is medial to the cavernous V2 segment throughout the cavernous sinus. Superior to the V2 is the ophthalmic nerve and the abducens nerve. Inferiorly, the posterior one-third of the V2 is related to the V3, while the posterior two-thirds is related to the dural floor of the sinus.

From an endoscopic transmaxillary perspective, the V2 can be followed proximally to expose its cavernous segment after drilling the pterygoid process of the sphenoid bone and the middle fossa floor to open the foramen rotundum. After the foramen is opened, the dural covering of the nerve becomes a part of the anterior wall of the cavernous sinus. Kerrison rongeurs are used to expose the anterior wall of the cavernous sinus, which opens to track the V2 within the sinus, thus enabling access to the sinus and its contents (Fig. 10). For a better angular view, a $30^{\circ}$ or a $45^{\circ}$ endoscope can be used; this provides an excellent view of the interior of the sinus. Identifying the foramen rotundum as an avenue to the cavernous sinus is useful in cases of cavernous sinus lesions.

\section{Illustrative Case}

Somatostatin therapy and cabergoline had failed in a 67-year-old man who had a recurrent growth hormoneproducing pituitary adenoma (Fig. 11) refractory to multimodal treatment and a history of multiple transsphenoidal resections and maximal radiotherapy. The cavernous sinus

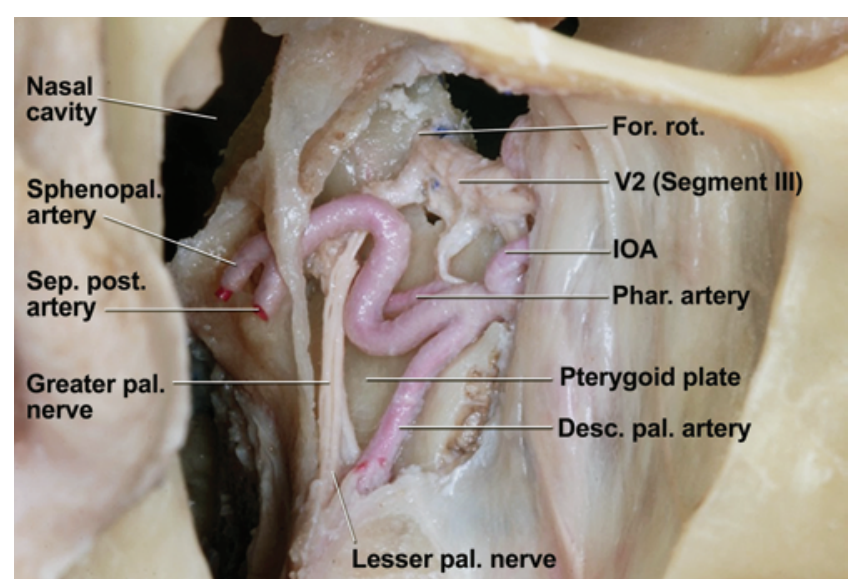

FIG. 6. The pterygopalatine segment (Segment III) of the left ION/maxillary nerve (V2) complex, anterior to posterior view. The posterior wall of the maxillary sinus has been removed to expose the pterygopalatine fossa, which contains the internal maxillary artery, the pterygopalatine ganglion, and the maxillary nerve. The distal branches of the maxillary artery are the internal maxillary, the descending palatine (Desc. pal.) artery, the IOA, and the sphenopalatine (Sphenopal.) artery. The third segment of the maxillary nerve, the pterygopalatine segment (Segment III), is located between the foramen rotundum (For. rot.) and the inferior orbital fissure . pal. $=$ palatine; Phar. $=$ pharyngeal; Sep. post. $=$ septal posterior. Reproduced with permission from the Rhoton Collection (http://rhoton.ineurodb.org/), CC BY-NC-SA 4.0 (http://creativecommons. org/licenses/by-nc-sa/4.0/). Figure is available in color online only.

portion of the tumor was enlarging despite medical treatment. He had baseline left V2 and V3 facial numbness and ophthalmoplegia. An endonasal transmaxillary transpterygoid approach was presented as an option for palliative debulking of the cavernous sinus tumor to avoid a craniotomy and brain retraction. Another reasonable surgical option was a cranial extradural transcavernous approach. There was no expectation that surgery would yield biochemical remission of the acromegaly. In this case, a complete medial maxillectomy was performed to widely expose the maxillary sinus. The ION was identified in the

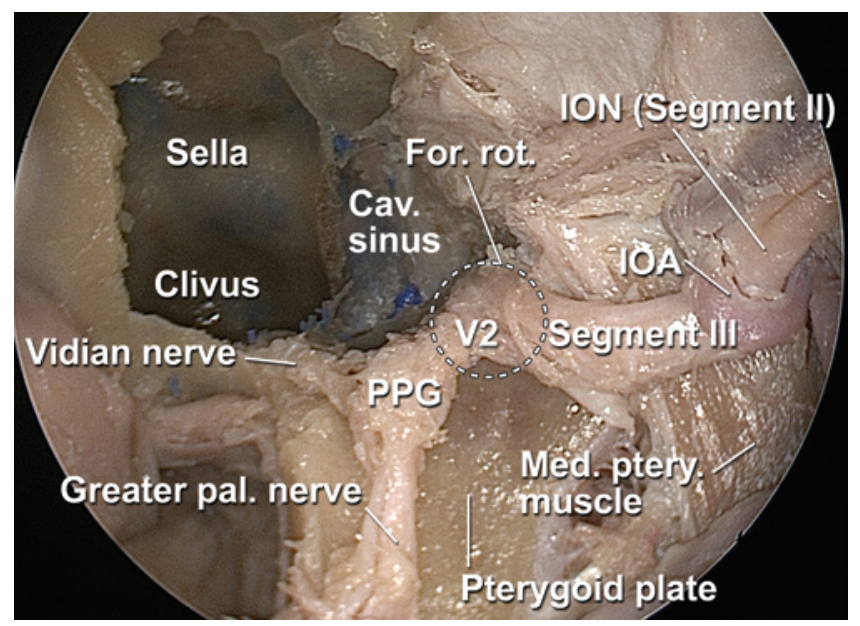

FIG. 7. Magnified endoscopic endonasal view of the left pterygopalatine fossa via a $0^{\circ}$ endoscope. Dashed circle indicates the foramen rotundum (For. rot.). Cav. = cavernous; pal. = palatine; Med. ptery. = medial pterygoid; PPG = pterygopalatine ganglion; V2 = maxillary nerve. Figure is available in color online only. 


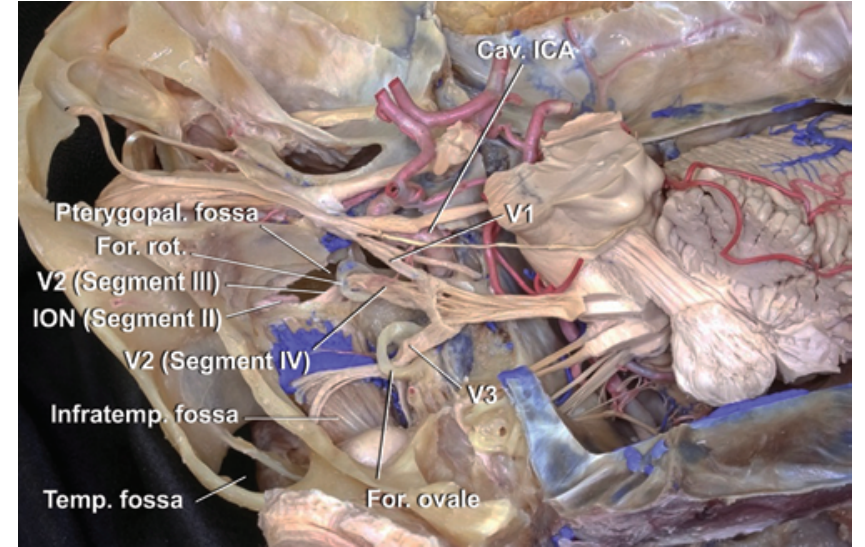

FIG. 8. The cavernous segment (Segment IV) of the maxillary nerve (V2). Lateral view of the left middle fossae: the greater wing of the sphenoid bone has been removed to expose the infratemporal (Infratemp.) fossa, which contains the pterygoid muscle, the venous plexus, and the third division of the mandibular nerve (V3). The first division of the trigeminal nerve (ophthalmic nerve [V1]) passes through the superior orbital fissure, the second division of the trigeminal nerve (maxillary nerve [V2]) passes through the foramen rotundum (For. rot.) to reach the pterygopalatine (Pterygopal.) fossa, and the mandibular nerve (V3) passes through the foramen (For.) ovale to reach the infratemporal (infratemp.) fossa. The infratemporal fossa is located lateral to the pterygopalatine fossa and medial to the temporal (Temp.) fossa. The cavernous segment of the maxillary nerve is located between the trigeminal ganglion and the foramen rotundum. Note the relationship between the cavernous (Cav.) ICA and the laterally positioned V2. Reproduced with permission from the Rhoton Collection (http://rhoton.ineurodb.org/), CC BY-NC-SA 4.0 (http://creativecommons.org/licenses/by-nc-sa/4.0/). Figure is available in color online only.

roof of the maxillary sinus. After the posterior wall of the maxillary sinus was removed, the pterygopalatine fossa contents and fat were identified by following the ION posteriorly. After the internal maxillary artery was sacrificed, the fossa contents were reflected laterally to expose the pterygoid wedge. The foramen rotundum was identified by

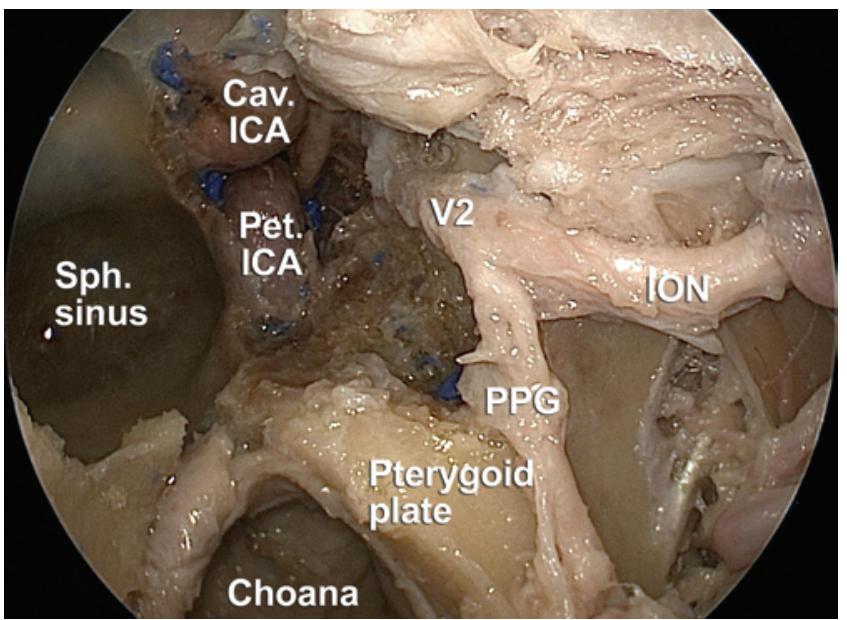

FIG. 9. Endoscopic endonasal view of the relationship between the left ION/maxillary nerve (V2) complex and the cavernous (Cav.) ICA and the anterior genu of the petrous (Pet.) ICA via a $0^{\circ}$ endoscope. The root of the pterygoid process of the sphenoid bone has been drilled to expose the lateral recess of the sphenoid (Sph.) sinus and the ICA. PPG = pterygopalatine ganglion. Figure is available in color online only.

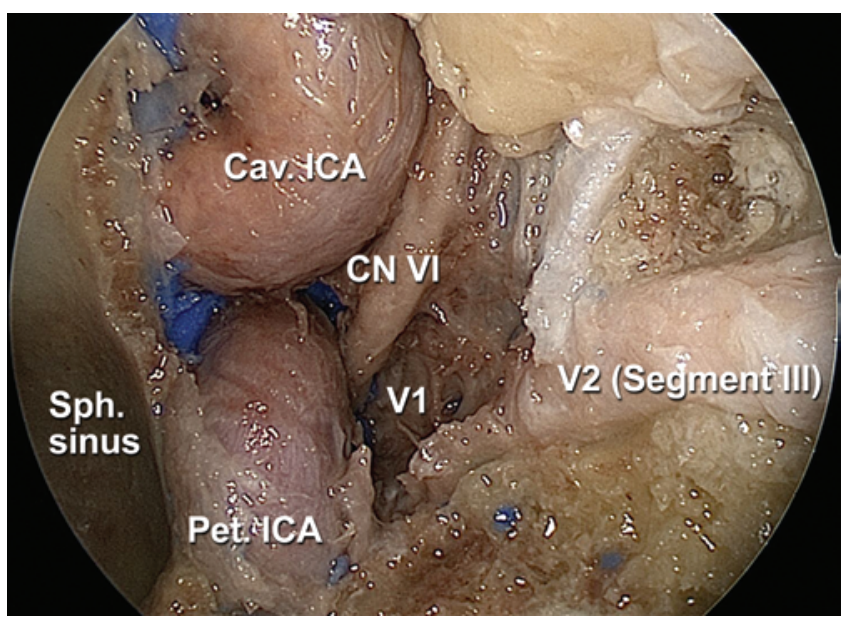

FIG. 10. Endoscopic endonasal view of the cavernous segment (Segment IV) of the ION/maxillary nerve (V2) complex via a $0^{\circ}$ endoscope. The pterygoid process of the sphenoid bone has been drilled to assist with access to the foramen rotundum, and the pterygopalatine ganglion is followed back as it forms the cavernous segment (Segment IV) of the maxillary nerve. Cav. = cavernous; $\mathrm{CN}$ VI = sixth cranial nerve; Pet. = petrous; Sph. = sphenoid; $\mathrm{V} 1=$ ophthalmic nerve. Figure is available in color online only.

tracing the ION posteriorly to the skull base. The sphenoid bone encompassing the foramen rotundum was drilled in an extradural fashion to provide a safe corridor superior and lateral to the ICA for entering the cavernous sinus and allowing for tumor resection. Aggressive tumor debulking was achieved with the expected residual tumor near the cavernous ICA.

\section{Discussion}

Surgically relevant intraoperative anatomical landmarks can be useful because of the complex regional anatomy encountered during ETMAs to the anterior skull base and because of the unfamiliar ventral endoscopic prospective. ${ }^{2,20,21}$ In the current study, we detailed the surgical anatomy of the ION/V2 complex as a reliable road map to the foramen rotundum, the cavernous sinus, the pterygopalatine fossa, and the anterolateral skull base during ETMAs. Upon entrance to the maxillary sinus, one can see that the ION (orbitomaxillary segment, or Segment II) is immediately visible through the thin superior wall of the maxillary sinus. With the ION/maxillary nerve as a landmark during dissection of the retromaxillary space, one can more easily discern the contents of the pterygopalatine fossa, the V2 and its branches, the internal maxillary artery, the vidian canal, the foramen rotundum, and the ICA. Our findings indicate that the ION is a potentially useful surgical landmark because it is easily identifiable early in the operative approach, it provides a pathway to the lateral cavernous sinus through the foramen rotundum, and it has a reliable relationship with other neurovascular structures of interest in ETMAs. The advantages of this ION classification are its simplicity, its relevance to endoscopic surgical anatomy in transmaxillary approaches, and its usefulness in identifying the skull base and the cavernous sinus.

Lesions within the anterolateral or retromaxillary spaces can be accessed endoscopically via a transmaxil- 


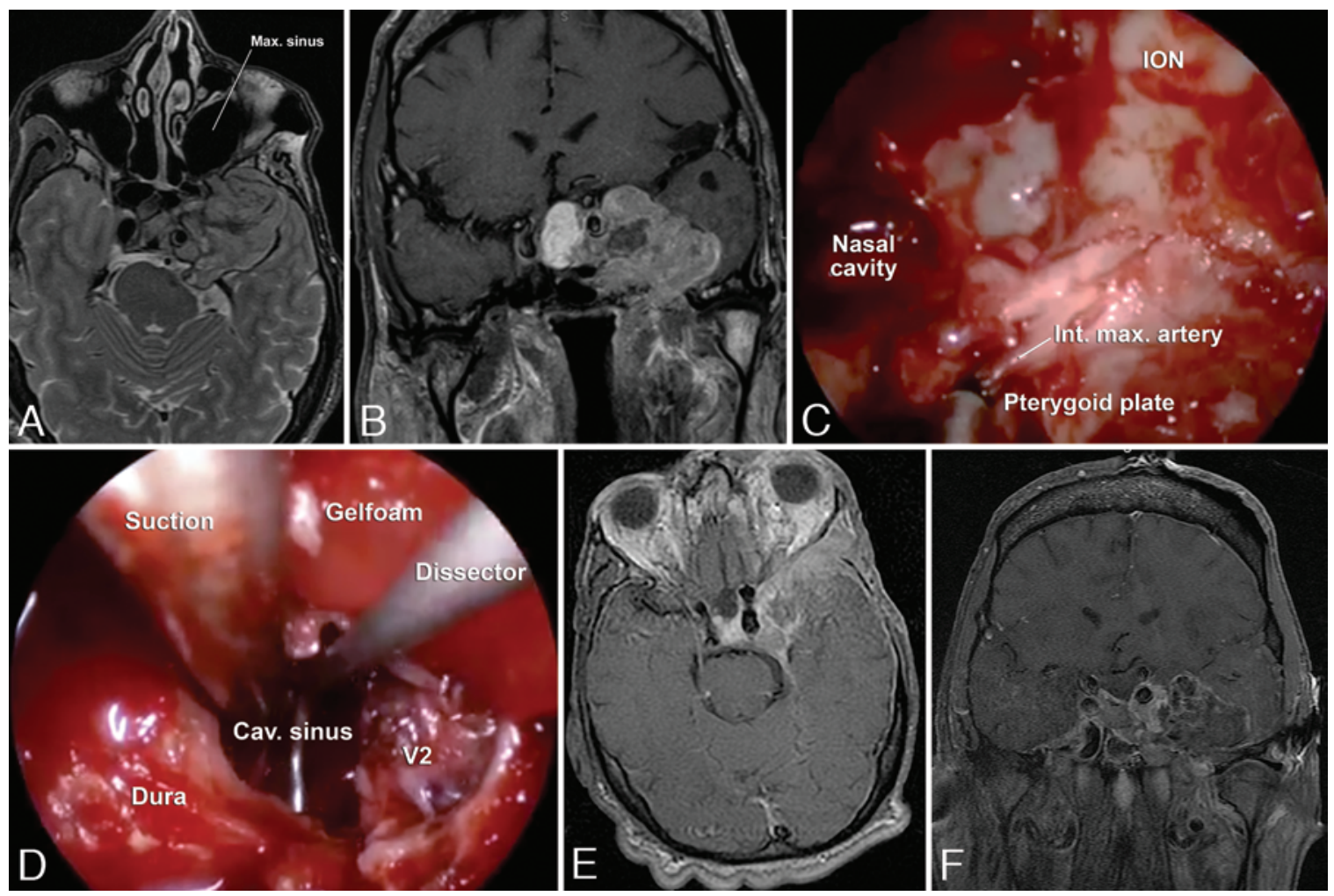

FIG. 11. Representative case of a 67-year-old man with acromegaly and recurrent adenoma in the left cavernous sinus after multiple transsphenoidal resections, radiotherapy, and medical therapies. Identifying the ION provided a landmark for entering the foramen rotundum through an endonasal endoscopic transmaxillary transpterygoid approach for palliative debulking of the tumor. Preoperative axial T2-weighted (A) and coronal T1-weighted (B) MR images demonstrating a large pituitary adenoma in the cavernous sinus. Intraoperative screenshot (C) demonstrating the left posterior maxillary sinus wall, with the ION impression visible in the bone. The posterior wall has been partially removed, demonstrating fascia of the pterygopalatine fossa. The bipolar forceps (bottom left) is grasping the internal maxillary (Int. max.) artery. The root of the pterygoid plate is labeled for orientation. Intraoperative screenshot (D) demonstrating how the tumor in the cavernous (Cav.) sinus has been entered by widening the foramen rotundum, reflecting the maxillary nerve (V2) laterally, and incising the dura. A suction tube and a ringed curette appear in the tumor cavity. Postoperative axial $(E)$ and coronal $(F)$ T1-weighted MR images demonstrating satisfactory tumor removal. Figure is available in color online only.

lary approach. ${ }^{23,25,28}$ A direct transmaxillary approach via a sublabial incision or a purely endonasal transmaxillary incision enables access to a wide range of anatomical targets within the infratemporal and pterygopalatine fossae. These targets include pathology located laterally from the temporomandibular joint and the zygoma to the cavernous sinus and sella medially, as well as lesions located within the orbital floor. ${ }^{8,12}$ Drilling of the pterygoid plates and the sphenoid bone allows access to deeper intracranial structures along the sphenoid bone and the deeper extracranial infratemporal structures. Stereotactic wand guidance can be an important means of assisting the surgeon in navigating the complex surgical corridor; however, a potential loss of accuracy remains during the course of the dissection. Ultimately, this approach requires the surgeon to develop a surgically relevant understanding of the anatomy within this complex region. In our description of the transmaxillary approach here, we have simplified the complex regional anatomy by dividing the ION/V2 complex into surgically relevant segments (Segments I-IV) for use as a road map to the anterior skull base. We hope that this surgically focused description will become another tool in the armamentarium of future endoscopic skull base surgeons.
Injury to the ICA is an uncommon yet potentially catastrophic complication, with reported occurrence rates of $0.4 \%-3.8 \%$ for traditional endoscopic transsphenoidal approaches. ${ }^{30}$ In transsphenoidal approaches, ICA injury most commonly occurs at the cavernous ICA. In extended skull base approaches, the highest risk occurs during drilling of the pterygoid process of the sphenoid bone to enter the foramen rotundum or during dissection along the posterior ethmoidal wall ${ }^{7}$ to access the retromaxillary space. Our anatomical dissection studies suggest that the ION can serve as a road map to the ICA, and an understanding of the anatomical relationship of the ION/V2 complex to the ICA can assist in early identification of the ICA to avoid injury to it. Following the nerve as it enters the foramen rotundum to become the V2 keeps the cavernous ICA medial and the petrous ICA inferior and posterior to the foramen rotundum. By hugging the medial aspect of the V2 during dissections, one can infer the location of the ICA. Furthermore, by identifying the foramen rotundum early in the dissection, one can note and remove the bone of the skull base in an extradural fashion. Early in our experience, we were often surprised by how quickly we happened upon the bone of the face of the cavernous 
sinus and the middle fossa. Others have demonstrated that the vidian nerve is a useful landmark for identifying the anterior genu of the ICA. ${ }^{26}$ We have found that using both the vidian canal and the ION/V2 complex as surgical landmarks for the ICA has improved our understanding of the ICA anatomy and has allowed us to make wider openings into the cavernous sinus.

Neurological complications can also occur because of injury to the ION, the pterygopalatine ganglion, and the maxillary and mandibular branches of the trigeminal nerve (V2 and V3), ${ }^{21}$ resulting in facial anesthesia or xerophthalmia. Preservation of the perineural sheath during dissection can avoid inadvertent injury to this structure. We propose that early identification of the ION will allow the surgeon to better delineate the anatomy of the pterygopalatine fossa and the infratemporal fossa, and thus to limit these potential complications.

Anatomical dissection studies have evolved from simple descriptive guides to surgically relevant guides. Smith and Laws noted that endoscopic classification schemes for skull base targets are critical for future generations of endoscopic surgeons. ${ }^{30}$ Given the surge in popularity of endoscopic skull base surgery, several authors have proposed surgically relevant anatomical classification schemes for various other surgical approaches and anatomical targets. ${ }^{3,11,15,22,30}$ For example, Labib et al.'s classification of the ICA serves as a practical guide for endoscopic surgeons. ${ }^{22}$ Similarly, Iaconetta et al. proposed a classification scheme for the trochlear nerve for transorbital approaches. ${ }^{15}$ Our hope is that the application of surgical landmarks like the one we developed and those created by others will be used in combination in clinical practice to improve the safety of endoscopic skull base surgery.

\section{Conclusions}

In this study, we examined the endoscopic anatomy of the ION/V2 complex relevant to transmaxillary approaches to the anterolateral skull base. We performed cadaveric dissections to study the anatomy of the ION and to develop a simple, surgically relevant road map to deep structures. Our findings suggest that the ION is an easily identifiable landmark that can be located early in the surgical exposure and then traced to the pterygopalatine fossa, the petrous and cavernous ICAs, and the cavernous sinus. We propose that the ION can be a useful surgical landmark to apply, along with other techniques, to guide surgeons.

\section{Acknowledgments}

Kaan Yagmurlu, MD, performed the anatomical dissections and took the dissection photographs.

\section{References}

1. Alfieri A, Jho HD, Schettino R, Tschabitscher M: Endoscopic endonasal approach to the pterygopalatine fossa: anatomic study. Neurosurgery 52:374-380, 2003

2. Aust MR, McCaffrey TV, Atkinson J: Transnasal endoscopic approach to the sella turcica. Am J Rhinol 12:283-287, 1998

3. Bouthillier A, van Loveren HR, Keller JT: Segments of the internal carotid artery: a new classification. Neurosurgery 38:425-433, 1996
4. Cappabianca P, Jane JA Jr, Souweidane M: Endoscopy in neurosurgery. Neurosurg Focus 30(4):Introduction, 2011

5. Cavallo LM, Cappabianca P, Messina A, Esposito F, Stella L, de Divitiis E, et al: The extended endoscopic endonasal approach to the clivus and cranio-vertebral junction: anatomical study. Childs Nerv Syst 23:665-671, 2007

6. Choi J, Park HS: The clinical anatomy of the maxillary artery in the pterygopalatine fossa. J Oral Maxillofac Surg 61:72-78, 2003

7. Dusick JR, Esposito F, Malkasian D, Kelly DF: Avoidance of carotid artery injuries in transsphenoidal surgery with the Doppler probe and micro-hook blades. Neurosurgery 60 (4 Suppl 2):322-329, 2007

8. Elhadi AM, Almefty KK, Mendes GA, Kalani MY, Nakaji P, Dru A, et al: Comparison of surgical freedom and area of exposure in three endoscopic transmaxillary approaches to the anterolateral cranial base. J Neurol Surg B Skull Base 75:346-353, 2014

9. Elhadi AM, Hardesty DA, Zaidi HA, Kalani MY, Nakaji P, White WL, et al: Evaluation of surgical freedom for microscopic and endoscopic transsphenoidal approaches to the sella. Neurosurgery 11 (Suppl 2):69-79, 2015

10. Elhadi AM, Zaidi HA, Hardesty DA, Williamson R, Cavallo C, Preul MC, et al: Malleable endoscope increases surgical freedom compared with a rigid endoscope in endoscopic endonasal approaches to the parasellar region. Neurosurgery 10 (Suppl 3):393-399, 2014

11. Gibo H, Lenkey C, Rhoton AL Jr: Microsurgical anatomy of the supraclinoid portion of the internal carotid artery. J Neurosurg 55:560-574, 1981

12. Gönül E, Erdogan E, Düz B, Timurkaynak E: Transmaxillary approach to the orbit: an anatomic study. Neurosurgery 53:935-942, 2003

13. Heilman CB, Shucart WA, Rebeiz EE: Endoscopic sphenoidotomy approach to the sella. Neurosurgery 41:602-607, 1997

14. Hofstetter CP, Singh A, Anand VK, Kacker A, Schwartz TH: The endoscopic, endonasal, transmaxillary transpterygoid approach to the pterygopalatine fossa, infratemporal fossa, petrous apex, and the Meckel cave. J Neurosurg 113:967-974, 2010

15. Iaconetta G, de Notaris M, Benet A, Rincon J, Cavallo LM, Prats-Galino A, et al: The trochlear nerve: microanatomic and endoscopic study. Neurosurg Rev 36:227-238, 2013

16. Jho HD, Carrau RL: Endoscopic endonasal transsphenoidal surgery: experience with 50 patients. J Neurosurg 87:44-51, 1997

17. Kassam A, Snyderman CH, Mintz A, Gardner P, Carrau RL: Expanded endonasal approach: the rostrocaudal axis. Part I. Crista galli to the sella turcica. Neurosurg Focus 19(1):E3, 2005

18. Kassam A, Snyderman CH, Mintz A, Gardner P, Carrau RL: Expanded endonasal approach: the rostrocaudal axis. Part II. Posterior clinoids to the foramen magnum. Neurosurg Focus 19(1):E4, 2005

19. Kassam AB, Gardner P, Snyderman C, Mintz A, Carrau R: Expanded endonasal approach: fully endoscopic, completely transnasal approach to the middle third of the clivus, petrous bone, middle cranial fossa, and infratemporal fossa. Neurosurg Focus 19(1):E6, 2005

20. Kassam AB, Gardner PA, Snyderman CH, Carrau RL, Mintz AH, Prevedello DM: Expanded endonasal approach, a fully endoscopic transnasal approach for the resection of midline suprasellar craniopharyngiomas: a new classification based on the infundibulum. J Neurosurg 108:715-728, 2008

21. Kassam AB, Prevedello DM, Carrau RL, Snyderman CH, Thomas A, Gardner P, et al: Endoscopic endonasal skull base surgery: analysis of complications in the authors' initial 800 patients. J Neurosurg 114:1544-1568, 2011 
22. Labib MA, Prevedello DM, Carrau R, Kerr EE, Naudy C, Abou Al-Shaar H, et al: A road map to the internal carotid artery in expanded endoscopic endonasal approaches to the ventral cranial base. Neurosurgery 10 (Suppl 3):448-471, 2014

23. Little AS, Nakaji P, Milligan J: Endoscopic endonasal transmaxillary approach and endoscopic sublabial transmaxillary approach: surgical decision-making and implications of the nasolacrimal duct. World Neurosurg 80:583-590, 2013

24. Messina A, Bruno MC, Decq P, Coste A, Cavallo LM, de Divittis E, et al: Pure endoscopic endonasal odontoidectomy: anatomical study. Neurosurg Rev 30:189-194, 2007

25. Ong BC, Gore PA, Donnellan MB, Kertesz T, Teo C: Endoscopic sublabial transmaxillary approach to the rostral middle fossa. Neurosurgery 62 (3 Suppl 1):30-37, 2008

26. Pinheiro-Neto CD, Fernandez-Miranda JC, Prevedello DM, Carrau RL, Gardner PA, Snyderman CH: Transposition of the Pterygopalatine Fossa during Endoscopic Endonasal Transpterygoid Approaches. J Neurol Surg B Skull Base 74:266-270, 2013

27. Resch KDM, Perneczky A: Endoscopic approaches to the suprasellar region: Anatomy and current clinical application. Adv Neurosurg 22:126-133, 1994

28. Robinson S, Patel N, Wormald PJ: Endoscopic management of benign tumors extending into the infratemporal fossa: a two-surgeon transnasal approach. Laryngoscope 115:18181822,2005

29. Sekhar LN, Nanda A, Sen CN, Snyderman CN, Janecka IP: The extended frontal approach to tumors of the anterior, middle, and posterior skull base. J Neurosurg 76:198-206, 1992

30. Smith TR, Laws ER Jr: Paradigm shift: the endoscopic carotid. World Neurosurg 83:157-159, 2015

31. Snyderman CH, Carrau RL, Kassam AB, Zanation A, Prevedello D, Gardner P, et al: Endoscopic skull base surgery: principles of endonasal oncological surgery. J Surg Oncol 97:658-664, 2008
32. Solari D, Magro F, Cappabianca P, Cavallo LM, Samii A, Esposito $\mathrm{F}$, et al: Anatomical study of the pterygopalatine fossa using an endoscopic endonasal approach: spatial relations and distances between surgical landmarks. J Neurosurg 106:157-163, 2007

33. Theodosopoulos PV, Guthikonda B, Brescia A, Keller JT, Zimmer LA: Endoscopic approach to the infratemporal fossa: anatomic study. Neurosurgery 66:196-203, 2010

34. Wilson DA, Williamson RW, Preul MC, Little AS: Comparative analysis of surgical freedom and angle of attack of two minimal-access endoscopic transmaxillary approaches to the anterolateral skull base. World Neurosurg 82:e487-e493, 2014

35. Yagmurlu K, Rhoton AL Jr, Tanriover N, Bennett JA: Threedimensional microsurgical anatomy and the safe entry zones of the brainstem. Neurosurgery 10 (Suppl 4):602-620, 2014

\section{Disclosures}

Dr. Little is an investor in Kogent Surgical.

\section{Author Contributions}

Conception and design: Little, Zaidi. Acquisition of data: Elhadi. Analysis and interpretation of data: Elhadi, Zaidi. Drafting the article: Elhadi, Zaidi. Critically revising the article: Little, Zaidi, Yagmurlu, Ahmed, Rhoton, Nakaji. Reviewed submitted version of manuscript: Little, Zaidi, Yagmurlu, Ahmed, Rhoton, Nakaji, Preul. Statistical analysis: Elhadi, Preul. Administrative/technical/ material support: Little. Study supervision: Little.

\section{Correspondence}

Andrew S. Little, c/o Neuroscience Publications, Barrow Neurological Institute, St. Joseph's Hospital and Medical Center, 350 W. Thomas Rd., Phoenix, AZ 85013.email: neuropub@ dignityhealth.org. 